\title{
One-dimensional classical adjoint SU(2) Coulomb Gas
}

\author{
Michael Engelhardt*† \\ Institut für theoretische Physik \\ Universität Tübingen \\ Auf der Morgenstelle 14 \\ 72076 Tübingen, Germany
}

()

\begin{abstract}
The equation of state of a one-dimensional classical nonrelativistic Coulomb gas of particles in the adjoint representation of SU(2) is given. The problem is solved both with and without sources in the fundamental representation at either end of the system. The gas exhibits confining properties at low densities and temperatures and deconfinement in the limit of high densities and temperatures. However, there is no phase transition to a regime where the string tension vanishes identically; true deconfinement only happens for infinite densities and temperatures. In the low density, low temperature limit, a new type of collective behavior is observed.
\end{abstract}

PACS: 05.70.Ce, 11.10.Kk, 11.15.-q

Keywords: Adjoint color sources, classical Coulomb gas

\section{INTRODUCTION}

Recently, there has been renewed interest in nonabelian classical Coulomb gases in one space dimension [1]- [3], in particular ones which contain particles in the adjoint representation of the gauge group. This is motivated by dimensional reduction arguments which map the gluonic fields of QCD in two space dimensions onto adjoint scalar fields in one space dimension 断. Such models are thus thought to contain some of the nontrivial gluonic dynamics, e.g. the degrees of freedom of the color flux string, of higher-dimensional Yang-Mills theories. While adjoint QCD is not exactly solvable even in one space dimension, some of these features have indeed been observed in approximate calculations exploiting the limit of a large number of colors $\mathrm{N}_{C}$ [4]- [8]. If one further specializes to the limit of heavy matter fields while retaining the large- $\mathrm{N}_{C}$ limit, it is possible to solve for the thermodynamic properties of the one-dimensional gas of adjoint particles [1]- [3]; one finds a deconfinement phase transition as a function of density or temperature [1]- 河], [7].

The purpose of the present note is to supplement this information with the thermodynamics of the classical nonrelativistic adjoint Coulomb gas in the opposite limit of only two colors. The thermodynamics of analogous systems containing abelian particles [9] or particles in the fundamental representation of $\mathrm{SU}(2)$ [10], [11] has been known for some time and it turns out that the case of adjoint particles can be solved with the same techniques as employed in [9], [11. Below, a pure adjoint Coulomb gas as well as an adjoint Coulomb gas in the presence of two charges in the fundamental representation of $\mathrm{SU}(2)$ are investigated. As is to be expected, the system exhibits confining properties at low densities and temperatures and deconfinement in the limit of high densities or temperatures. In contradistinction to the large- $\mathrm{N}_{C}$ case, the two regions are not separated by a phase transition; there is a continuous crossover between the two types of behavior. This is natural for a one-dimensional model with only a finite number of degrees of freedom at each space point. The most interesting feature is a new form of collective behavior at low densities and temperatures which is not observed for particles in the fundamental representation or abelian particles.

\section{THE MODEL}

In one space dimension, an array of color sources is connected by color strings which begin and end at sources and may overlap (note that there are no propagating gluon degrees of freedom in one space dimension). If one thinks of the array of sources as being built up one by one from one end to the other, then $n$ strings emanating from the first $k$

*email: engelm@pthp1.tphys.physik.uni-tuebingen.de

${ }^{\dagger}$ Supported by Deutsche Forschungsgemeinschaft under DFG Re 856 / 1-3 
sources corresponds to these sources being coupled to color spin $n / 2$ in the case of SU(2) color. Adding the $(k+1)$-th color source may change the number of color strings according to the possible couplings of the $(k+1)$-th source to the first $k$. E.g. in the case of $\mathrm{SU}(2)$ fundamental sources [10], [11], $n \pm 1$ strings may emanate from the $(k+1)$-th source; in the case of $\mathrm{SU}(2)$ adjoint sources, $n$ or $n \pm 2$ strings may emanate from the $(k+1)$-th source since the adjoint sources carry color spin one. In a physical system, the complete array of particles acting as sources must be coupled to a color singlet, which also implies that every different coupling scheme, i.e. string configuration, enters the partition function with unit combinatorial weight.

The potential energy resulting from the formation of the strings between the sources can be read off by observing that $n$ overlapping strings are associated with an energy density of $\epsilon_{n}=g^{2} n(n+2) / 8$ (cf. e.g. [12]). Therefore, given the positions $q_{i}$ of $N$ particles and the numbers $n_{i}^{C}$ of strings between the $i$-th and the $(i+1)$-th particle in a particular string configuration $C$, the associated potential energy is

$$
V_{C}=\frac{g^{2}}{8} \sum_{i=1}^{N} n_{i}^{C}\left(n_{i}^{C}+2\right)\left(q_{i+1}-q_{i}\right)
$$

where $n_{N}^{C}=0$. Also defining $n_{0}^{C}=0$, one can write this as

$$
V_{C}=\frac{g^{2}}{8} \sum_{i=1}^{N} q_{i}\left(\left(n_{i-1}^{C}+1\right)^{2}-\left(n_{i}^{C}+1\right)^{2}\right)
$$

For a system consisting purely of particles in the adjoint representation, the $n_{i}^{C}$ are subject to the constraints

$$
n_{0}^{C}=n_{N}^{C}=0 \quad n_{i}^{C} \geq 0 \quad\left|n_{i+1}^{C}-n_{i}^{C}\right| \in\{0, \pm 2\}
$$

The set of possible configurations $C$ is given by all sets $n_{i}^{C}, i=0, \ldots, N$, which satisfy the above constraints.

\section{THERMODYNAMICS}

The classical thermodynamics of a Coulomb gas of $N$ nonrelativistic adjoint particles 1 is most easily calculated in the canonical constant pressure ensemble [9] (the physical reason for this is discussed in [13]). In this ensemble, the Gibbs free energy after the trivial integrations over the canonical momenta is given by

$$
G=-\frac{1}{\beta} \ln \left[\left(\frac{2 m \pi}{\beta}\right)^{N / 2} Q\right]
$$

with the configuration integral

$$
\begin{aligned}
Q & =\sum_{C} \int_{0}^{\infty} d q_{N} \int_{0}^{q_{N}} d q_{N-1} \ldots \int_{0}^{q_{2}} d q_{1} e^{-\beta\left(P q_{N}+V_{C}\right)} \\
& =\sum_{C} Q_{C}
\end{aligned}
$$

where $P$ denotes the pressure. Due to the linear dependence of $V_{C}$ on the $q_{i}$, the integrations in (5) can all be carried out using the properties of the Laplace transform [13], yielding

$$
Q_{C}=\left(\frac{8}{\beta g^{2}}\right)^{N}(\gamma+1) \frac{1}{\prod_{i=0}^{N}\left(\gamma+\left(n_{i}^{C}+1\right)^{2}\right)}
$$

where the abbreviation $P=g^{2}(\gamma+1) / 8$ was introduced. It remains to evaluate the sum over configurations $C$. In order to accomplish this, one defines a cluster at level $r$ as a sequence of factors in (7) such that $n_{i}^{C} \geq r$ for all $n_{i}^{C}$ in the sequence, but $n_{i}^{C}<r$ for the two factors adjacent on either side. This in particular implies $n_{i}^{C}=r$ for the first

\footnotetext{
${ }^{1}$ The realm of validity of such a treatment is discussed in 11 .
} 
and the last factor in the sequence; the value $r$ is not excluded for other $n_{i}^{C}$ in between. Such a cluster thus physically corresponds to a sequence of interparticle spacings covered by $r$ or more string: Then one can define $G_{n}^{r}$ to be the sum over all clusters at level $r$ involving exactly $n$ factors. Ultimately, one is interested in $G_{N+1}^{0}=\left(\beta g^{2} / 8\right)^{N} Q /(\gamma+1)$.

These cluster sums are convenient in that one can give a recursion relation between the sums at different levels. Excluding for the moment the case of level $r=0$, one can characterize a cluster at level $r$ of length $n$ by the number $k$ of clusters at level $r+2$ it contains (this is at most the integer part of $(n-1) / 2)$, and the number $m$ of factors $\left(\gamma+(r+1)^{2}\right)$ present, i.e. the number of spacings in the cluster covered by exactly $r$ strings. This latter number is at least $k+1$ and at most $n-k$. Now, given $k$ and $m$, one must have at least one factor $\left(\gamma+(r+1)^{2}\right)$ between each pair of clusters at level $r+2$ present, and one at each end of the cluster at level $r$ under consideration. Thus, $k+1$ of the $m$ factors $\left(\gamma+(r+1)^{2}\right)$ are fixed, but the other $m-k-1$ factors can be arbitrarily distributed among the $k+1$ spaces between the clusters at level $r+2$ or at the two ends. The number of ways of doing this is $(m-1) ! /(k !(m-1-k) !)$. Finally, one must specify the lengths of the $k$ clusters at level $r+2$ subject to the constraint that the sum of these lengths must total $n-m$. Therefore, one has the recursion relation

$$
G_{n}^{r}=\frac{1}{\left(\gamma+(r+1)^{2}\right)^{n}}+\sum_{k=1}^{[(n-1) / 2]} \sum_{m=k+1}^{n-k}\left(\begin{array}{c}
m-1 \\
k
\end{array}\right) \frac{1}{\left(\gamma+(r+1)^{2}\right)^{m}} \sum_{i_{1}=1}^{\infty} \ldots \sum_{i_{k}=1}^{\infty} \delta_{i_{1}+\ldots+i_{k}, n-m} G_{i_{1}}^{r+2} \ldots G_{i_{k}}^{r+2}
$$

where the $k=0$ term has been written explicitly. This recursion relation can be simplified by going to the corresponding generating function

$$
G^{r}(z)=\sum_{n=1}^{\infty} G_{n}^{r} z^{n}
$$

Treating the $n=1$ and $n=2$ terms separately and rearranging summations,

$$
\sum_{n=3}^{\infty} \sum_{k=1}^{[(n-1) / 2]} \sum_{m=k+1}^{n-k}=\sum_{k=1}^{\infty} \sum_{m=k+1}^{\infty} \sum_{n=m+k}^{\infty}
$$

the Kronecker- $\delta$ constraint in (8) becomes trivial and one arrives at

$$
G^{r}(z)=\frac{1}{\left(\gamma+(r+1)^{2}\right) / z-1-G^{r+2}(z)}
$$

As mentioned above, the case $r=0$ must be treated separately. One cannot have consecutive factors $(\gamma+1)$ in a cluster at level zero, i.e. consecutive interparticle spacings with no strings, since color spin zero cannot be coupled with color spin one back to color spin zero. Physically, this means that the adjoint particles are confined Therefore, one has the constraint

$$
m=k+1
$$

and no $k=0$ term (one may set $G_{1}^{0}=G_{2}^{0}=0$; these quantities are anyway irrelevant). Proceeding in complete analogy to above, one arrives at

$$
G^{0}(z)=\frac{1}{(\gamma+1) / z-G^{2}(z)}-\frac{z}{\gamma+1}
$$

Now, one can reextract the $(N+1)$-th Taylor coefficient $G_{N+1}^{0}$ using

$$
G_{N+1}^{0}=\frac{1}{2 \pi i} \oint d z \frac{G^{0}(z)}{z^{N+2}}
$$

By blowing up the integration contour to infinity, one picks up contributions from all the poles $\alpha_{i}$ of $G^{0}(z)$,

\footnotetext{
${ }^{2}$ Note that the factors containing $n_{0}^{C}$ and $n_{N}^{C}$ have been added by hand to the original $N-1$ physical spacings.

${ }^{3}$ Note that this is not the case anymore in a higher number of space dimensions, where a single adjoint particle can form a color singlet bound state with a gluon.
} 


$$
G_{N+1}^{0}=-\sum_{i} \operatorname{res}_{\alpha_{i}} \frac{G^{0}(z)}{z^{N+2}}
$$

but for $N \rightarrow \infty$, this sum is dominated by the pole closest to the origin $\alpha_{1}$,

$$
\lim _{N \rightarrow \infty} \ln G_{N+1}^{0}=-N \ln \alpha_{1}+O(1)
$$

which implies for the Gibbs free energy

$$
G=-\frac{N}{\beta}\left(\frac{1}{2} \ln \frac{2 m \pi}{\beta}+\ln \frac{8}{\beta g^{2}}-\ln \alpha_{1}\right)
$$

In view of (13) and (11), $\alpha_{1}$ is the smallest solution of

$$
\frac{\gamma+1}{z}=G^{2}(z)=\frac{1}{(\gamma+9) / z-1-\frac{1}{(\gamma+25) / z-1-\frac{1}{(\gamma+49) / z-1-\ldots}}}
$$

For not too high pressures $P=g^{2}(\gamma+1) / 8$, one can easily obtain $\alpha_{1}$ numerically from this equation, yielding the exact Gibbs free energy by inserting in (17). For high pressures, it is advantageous to use an asymptotic expansion for $\alpha_{1}$, which will now be obtained along with an explicit low pressure expansion. By denoting $a=z-\gamma$ and $V_{m}=\left(a-m^{2}\right) / z$, (18) takes the form

$$
0=V_{1}-1-\frac{1}{V_{3}-\frac{1}{V_{5}-\frac{1}{V_{7}-\ldots}}}
$$

which is just the relation determining the characteristic values corresponding to even periodic solutions of odd order of the Mathieu equation, denoted in [14 as $a_{2 r+1}(z)$. Consequently, if $\gamma$ is given, then $\alpha_{1}$ is the lowest solution of $a_{2 r+1}(z)=z-\gamma$. The lowest solution occurs for $r=0$, i.e. $\gamma=z-a_{1}(z)$. Now, the limiting behavior of $a_{1}(z)$ is known and gives, translated back to $\alpha_{1}$ and $P$,

$$
\begin{aligned}
& \frac{8 P}{g^{2}}=\frac{1}{8} \alpha_{1}^{2}+\frac{1}{64} \alpha_{1}^{3}+\frac{1}{1536} \alpha_{1}^{4}-\frac{11}{36864} \alpha_{1}^{5}-\frac{49}{589824} \alpha_{1}^{6}+\ldots \quad \text { for } \alpha_{1} \rightarrow 0 \\
& \frac{8 P}{g^{2}}=3 \alpha_{1}-6 \alpha_{1}^{1 / 2}+\frac{9}{4}+\frac{3}{32} \alpha_{1}^{-1 / 2}+\frac{45}{256} \alpha_{1}^{-1}+\ldots \quad \text { for } \alpha_{1} \rightarrow \infty
\end{aligned}
$$

It is more intuitive to consider the equation of state, which can be obtained by inverting (20) and (21), inserting in

$$
V=\left.\frac{\partial G}{\partial P}\right|_{T} \quad \Rightarrow \quad \rho T=\frac{N T}{V}=\frac{\alpha_{1}}{\left.\left(\partial \alpha_{1} / \partial P\right)\right|_{T}}
$$

and again inverting the resulting expansions. In this way one arrives at

$$
\begin{aligned}
& P=\rho T\left(\frac{1}{2}-\frac{\sqrt{2}}{8}\left(\frac{\rho T}{g^{2}}\right)^{1 / 2}+\frac{19}{96} \frac{\rho T}{g^{2}}+\frac{73 \sqrt{2}}{1536}\left(\frac{\rho T}{g^{2}}\right)^{3 / 2}-\frac{121}{1152}\left(\frac{\rho T}{g^{2}}\right)^{2}+\ldots\right) \\
& P=\rho T\left(1-\frac{\sqrt{6}}{4}\left(\frac{g^{2}}{\rho T}\right)^{1 / 2}+\frac{3}{32} \frac{g^{2}}{\rho T}-\frac{15 \sqrt{6}}{2048}\left(\frac{g^{2}}{\rho T}\right)^{3 / 2}+\frac{99}{8192}\left(\frac{g^{2}}{\rho T}\right)^{2}+\ldots\right)
\end{aligned}
$$

The reason for the pressure only depending on the combination $\rho T$ is discussed in 13 . The exact equation of state, as obtained from (22) in conjunction with (18), is plotted in Fig. 1, compared with the case of particles in the fundamental representation of $\mathrm{SU}(2)$ [10, 11.

\footnotetext{
${ }^{4}$ Note that the residue there is negative, since $G^{0}(z)$ rises monotonously on the real axis as it approaches $\alpha_{1}$.
} 
Before discussing these results, it is instructive to repeat the above calculation for the case that a particle in the fundamental representation of $\mathrm{SU}(2)$ is positioned at either end of the system. This modifies the possible set of color string configurations as follows: One starts with one color string at one end of the array of adjoint particles and ends again with one color string at the other end, i.e. $n_{0}^{C}=n_{N}^{C}=1$. One can never have less than one string, i.e. $n_{i}^{C} \geq 1$. In all other ways, however, the combinatorics remains the same; one has the same set of configurations except that the number of strings is augmented by one everywhere. Therefore, one arrives again at the recursion relation (8), and consequently also (11), except that the index $r$ now runs over all odd numbers. Note also that at the lowest level, $r=1$, one has no special constraint analogous to 12 for the case $r=0$; one can couple one string, corresponding to color spin one-half, with a color spin one adjoint particle back to color spin one-half. Physically, in the color electric background generated between a pair of separated fundamental sources, adjoint particles are deconfined. By contrast, in the vacuum, adjoint particles must combine to form color singlet clusters made up of two or more particles.

The thermodynamic behavior of the system is now determined in complete analogy to above by the pole $\beta_{1}$ of $G^{1}(z)$ nearest to the origin, i.e. the lowest solution of

$$
\frac{\gamma+4}{z}-1=\frac{1}{(\gamma+16) / z-1-\frac{1}{(\gamma+36) / z-1-\frac{1}{(\gamma+64) / z-1-\ldots}}}
$$

With the same abbreviations as above, this translates to the equation

$$
0=V_{2}-\frac{1}{V_{4}-\frac{1}{V_{6}-\frac{1}{V_{8}-\ldots}}}
$$

determining the characteristic values corresponding to odd periodic solutions of even order of the Mathieu equation, denoted in 14] as $b_{2 r}(z)$. For given $\gamma$, one thus obtains $\beta_{1}$ as the lowest solution of $b_{2 r}(z)=z-\gamma$, which occurs for $r=1$, i.e. $\gamma=z-b_{2}(z)$. Using again the known limiting behavior of $b_{2}(z)$, one arrives at the following virial expansion of the equation of state,

$$
P=-\frac{3}{8} g^{2}+\rho T\left(1-\frac{2}{3} \frac{\rho T}{g^{2}}+\frac{16}{9}\left(\frac{\rho T}{g^{2}}\right)^{2}-\frac{145}{27}\left(\frac{\rho T}{g^{2}}\right)^{3}+\frac{1472}{81}\left(\frac{\rho T}{g^{2}}\right)^{4}+\ldots\right)
$$

On the other hand, for large densities $\rho$, one has the same expansion as in the case without fundamental sources (cf. eq. (24)), since the characteristic values of the Mathieu equation $b_{2}$ and $a_{1}$ have the same asymptotic expansion for large arguments $z$. Note however that for any finite $z, a_{1}$ and $b_{2}$ differ [14] and

$$
b_{2}-a_{1} \sim 2^{9} \sqrt{2 / \pi} z^{5 / 4} e^{-4 \sqrt{z}} \quad \text { for } z \rightarrow \infty
$$

which of course does not show up in a $1 / z$-expansion.

\section{DISCUSSION}

The thermodynamic behavior displayed by the one-dimensional classical Coulomb gas of particles in the adjoint representation of $\mathrm{SU}(2)$ can be interpreted as follows: In the low density, low temperature limit, the equation of state (23) yields the pressure corresponding to a system of $N / 2$ particles, since the $N$ adjoint constituents are confined and pair off to form color singlet conglomerates in order to minimize the amount of energy invested into color strings. The behavior near this limit cannot be described by the usual virial expansion in powers of the density $\rho$, as happens e.g. for particles in the fundamental representation [10], [11]; instead, one obtains an expansion in $\sqrt{\rho T / g^{2}}$. This is a signature for collective behavior under participation of arbitrarily many particles; any cluster approximation taking into account only interactions between a finite number of particles at a time will necessarily lead to an expansion in powers of $\rho$. The origin of this collective behavior is not difficult to pinpoint: Adjoint particles have the possibility of forming multi-particle clusters with only two color electric strings extending over their entire length. These clusters are quite cheap in terms of potential energy and provide a high level of degeneracy already in the low-lying spectrum. Indeed, one can verify that by taking into account only the aforementioned configurations, i.e. approximating $G_{n}^{2}=1 /(\gamma+9)^{n}$, leading to $G^{2}(z)=z /(\gamma+9-z)$ in $(18)$, one reproduces exactly the first two 
terms in (23). Such an effect does not occur for fundamental sources, since for these, string configurations of the type described above are impossible. These configurations are analogous to the states which have been observed in [i][8] to proliferate in the spectrum of large- $\mathrm{N}_{C}$ adjoint $\mathrm{QCD}_{1+1}$, generating an exponentially rising density of states leading to a Hagedorn limiting temperature. One should realize that in the large- $\mathrm{N}_{C}$ limit, the effect of these states is greatly enhanced by the fact that the color strings are labeled by an additional infinity of quantum numbers compared with the present case of $\mathrm{SU}(2)$. For $\mathrm{SU}(2)$, there is no sharp phase transition; the crossover to the high density, high temperature regime is smooth, as evidenced in Fig. 1. This is to be expected on general grounds in a one-dimensional model; only in the limit of infinitely many colors, where the infinity of degrees of freedom at each space point acts like an extra dimension, can one obtain a genuine deconfinement phase transition [1]- 3], [7].

Nevertheless, in the present case of $\mathrm{SU}(2)$ color, the thermodynamic behavior in the low density, low temperature limit is dominated by the effect of arbitrarily large multi-particle clusters which only contain two color strings along their entire length; as is to be expected, the formation of these clusters initially leads to a lowering of the pressure as compared with a gas of $N / 2$ particles composed of pairs of adjoint constituents. Only at higher densities or temperatures does the equation of state turn around to approach the high density, high temperature limit, cf. eq. (24), in which the system behaves like an ideal gas of the $N$ adjoint particles. In the presence of many nearby partners, an adjoint particle may propagate on its own over large distances without having to invest appreciable energy. The leading behavior away from this deconfined limit is nonanalytic in the string tension, namely proportional to $\sqrt{g^{2} /(\rho T)}$. This signals the presence of collective plasma excitations in the medium in analogy to the Debye-Hückel law of electrodynamics. In this respect, the gas of adjoint particles behaves no different from the gas of particles in the fundamental representation [10], [11].

Turning now to the analysis of the case where a fundamental particle is placed at either end of the system, one observes a negative pressure $P=-3 g^{2} / 8$ in the low density, low temperature limit, cf. eq. (27). This is simply the force needed to keep the two fundamental particles apart such that the system retains the desired one-dimensional volume $V$ (note that in one dimension, a pressure corresponds to a force since there is no transverse area to divide by). The value $3 g^{2} / 8$ is precisely the string tension associated with the one color string extending between the fundamental sources. If one did not exert this force, the system would strive to contract to smaller volumes until the pressure of the adjoint particles equalized the confining linear force between the two fundamental sources. The leading behavior away from this limit corresponds to the pressure generated by the $N$ adjoint deconfined particles. This stands in accordance with the remark already made further above, that adjoint particles are deconfined in the chromoelectric background generated by the two fundamental sources, in contradistinction to the vacuum, where they are confined and must form color singlet clusters. For this same reason, also the collective effects stemming from the formation of multi-particle clusters present in the vacuum are absent in the chromoelectric background; the low density, low temperature expansion of the equation of state takes the usual form containing only powers of $\rho$, cf. eq. (27).

On the other hand, in the high density, high temperature limit, the pressure exerted by the system with two fundamental sources at the ends is the same as the one exerted by the system without these sources, to all orders in $\sqrt{g^{2} /(\rho T)}$. Therefore, in this limit, there is no vestige of the original confining force between the two fundamental sources; the force is entirely screened and the sources are deconfined. However, the expansion in $\sqrt{g^{2} /(\rho T)}$ is merely asymptotic; for any finite value of $\rho T / g^{2}$, the pressure of the system with fundamental sources is lower by an amount vanishing exponentially in $\sqrt{\rho T / g^{2}}$ for large $\rho T / g^{2}$ in view of eq. (28). In other words, the string tension between fundamental sources in the presence of the medium of adjoint particles falls off exponentially for large $\rho T / g^{2}$. In particular, there is no entire regime in $\rho T / g^{2}$ where the string tension vanishes identically and which thus could be interpreted as a truly deconfined phase.

[1] G.W.Semenoff, O.Tirkkonen and K.Zarembo, Phys. Rev. Lett. 77 (1996) 2174

[2] G.W.Semenoff and K.Zarembo, Nucl. Phys. B480 (1996) 317

[3] C.R.Gattringer, L.D.Paniak and G.W.Semenoff, preprint hep-th/9612030

[4] K.Demeterfi, I.Klebanov and G.Bhanot, Nucl. Phys. B418 (1994) 15

[5] S.Dalley and I.Klebanov, Phys. Rev. D 47 (1993) 2517

[6] G.Bhanot, K.Demeterfi and I.Klebanov, Phys. Rev. D 48 (1993) 4980

[7] D.Kutasov, Nucl. Phys. B414 (1994) 33

[8] I.Kogan and A.R.Zhitnitsky, Nucl. Phys. B465 (1996) 99

[9] A.Lenard, J. Math. Phys. 2 (1961) 682

[10] Y.Nambu, B.Bambah and M.Gross, Phys. Rev. D 26 (1982) 2875 
[11] M.Engelhardt, Phys. Lett. B345 (1995) 379

[12] M.Engelhardt and B.Schreiber, Z. Phys. A 351 (1995) 71

[13] M.Engelhardt, Phys. Rev. C 50 (1994) 3094

[14] M.Abramowitz, I.A.Stegun, eds., Handbook of Mathematical Functions (Dover, New York, 1965)

FIG. 1. Equation of state of the one-dimensional classical nonrelativistic Coulomb gas of particles in the adjoint representation of $\mathrm{SU}(2)$ (solid line) and in the fundamental representation of $\mathrm{SU}(2)$ (dashed). 


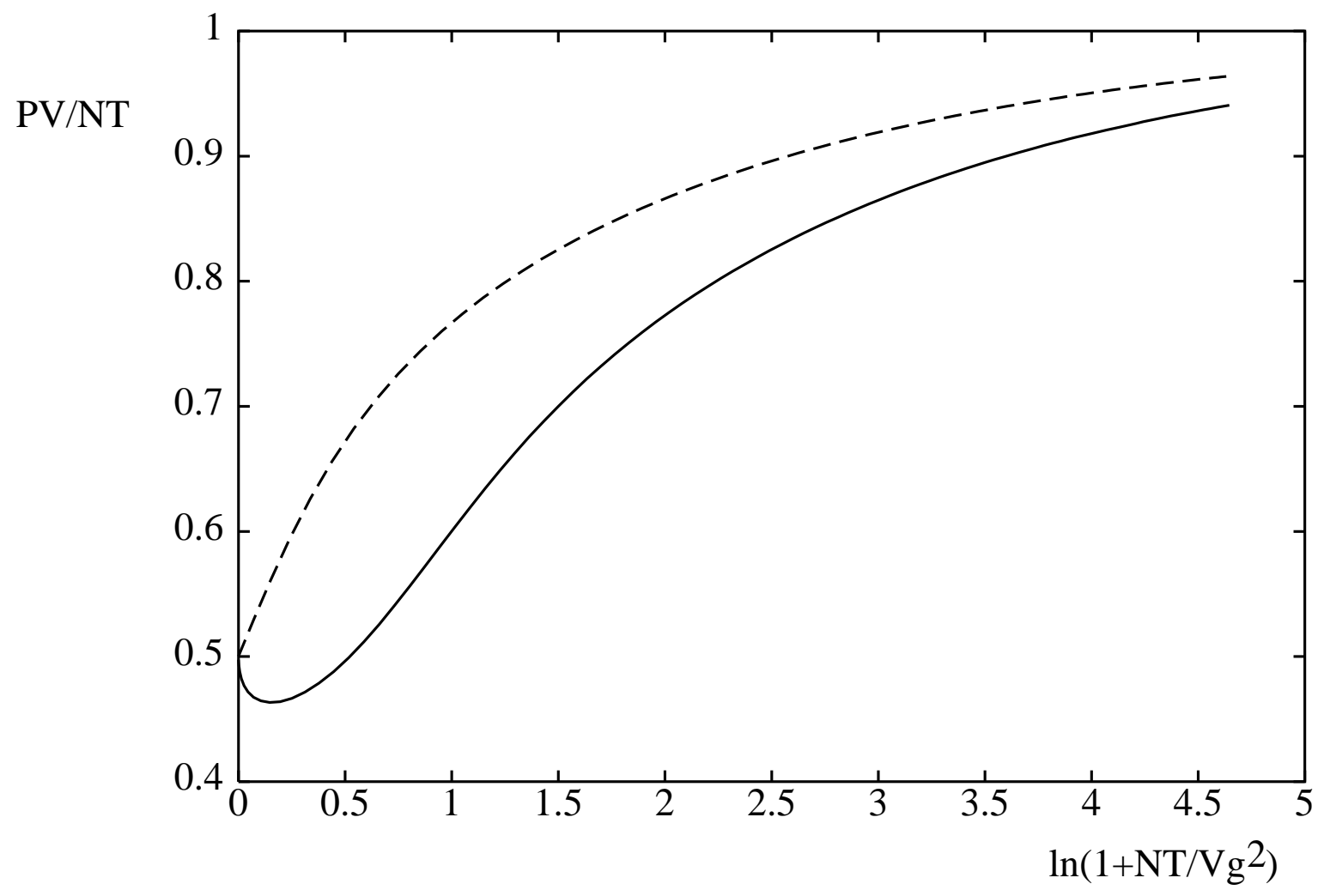

M. Engelhardt, Fig. 1 